\title{
Capillary Discharge Parameter Assessment for X-ray Laser Pumping
}

\author{
J. Hübner
}

\begin{abstract}
This paper assigns optimum capillary discharge characteristics with respect to reaching the maximum emission gain on wavelength $l=18.2 \mathrm{~nm}$ and corresponding to Balmer $\alpha$ transition H-like carbon. The computer modelling of the capillary discharge evolution is carried out using the NPINCH programme, using a one-dimensional physical model based on MHD equations. The information about the capillary discharge evolution is processed in FLY, FLYPAPER, FLYSPEC programmes, enabling the population to be modelled on specific levels during capillary discharge.
\end{abstract}

Keywords: capillary discharge, X-ray laser, MHD (magnetohydrodynamic) simulation, population inversion, gain optimalization, carbon.

\section{Introduction}

Non-stationary plasma of a fast capillary electrical discharge was studied as a potential active medium for a soft X-ray laser. The aim was to achieve an optimum set of characteristics for lasing at $18.2 \mathrm{~nm}$ wavelength H-like carbon $\mathrm{C}^{5+}$ in an alumina capillary. Optimum in the sense of reaching a maximum gain value for each set of parameters.

Capillary pinch dynamics is determined by many selected parameters: capillary geometry (radius and capillary length), the substance of the capillary, the initial filling pressure, and the electric current time dependance. This dependance, in particular, is given by an electric circuit which is joined to the capillary.

A capillary discharge Z-pinch acting as a medium for a soft X-ray laser uses ASE, the "Amplified Spontaneous Emission" effect, and electron-collisional recombination pumping. The main variable for ASE is the gain, and the most important goal of this paper is to find specific parameters of a capillary to obtain the maximum gain.

Electron-collisional recombination, sometimes referred to as "three body" recombination, is the inverse process to electron-collisional ionization from excited levels. The combined recombination and radiative downward cascading process is illustrated by the equation

$$
X_{o}^{(i+1)+}+2 e \rightarrow X_{n}^{i+}+e \rightarrow X_{u}^{i+}+e
$$

It is assumed that the ions exist plentifully in the initial state, which is one stage of ionization higher than that of the lasing ions. Note that this is not a self-contained population-replenishment scheme. Hence, replenishment depends upon further reionization, which is provided in our case by the release of the next current pulse.

It was assumed that ablation from inner walls has only an imperceptible effect, and so it was not in- cluded in the calculations. The capillary discharge plasma quantities were calculated by means of the NPINCH [2] code under the one-dimensional, twotemperature, one-fluid MHD approximation. The output data was then processed by means of the FLY code, which enables the creation of a history file of the population on the individual levels along the capillary axis. On the basis of the populations history, the gain and the gain history can be calculated.

\section{Optimization}

Optimization itself involves searching the maximum in the four-dimensional space of the parameters. Two parameters relate to the current-impulse shape, the third parameter is initial density (or pressure), and the fourth is the radius of the capillary.

The right choice in the range of parameters is of fundamental importance. The results of our study should be instrumental for experimenters in the CAPEX-U [4] project. The range of parameters therefore has to be related to their experimental equipment, with the view to verifying the results. Consequently, the choice of the first parameter was obvious because an alumina capillary $r=1.6 \mathrm{~mm}$ in radius is used in CAPEX-U. The choice of the current pulse had to coincide with the source in CAPEX-U. The electric current in a capillary was fitted from measuring by function.

$$
I_{f i t}=I_{0} \sin \left(\frac{\pi t}{2 t_{0}}\right) \exp \left(-\frac{t}{t_{1}}\right)
$$

The chosen parameters are therefore associated with equation 2: current peak value $I_{\max }$ and $\mathrm{d} I / \mathrm{d} t_{\mid t=0}$. Two cases were calculated $I_{\max } \approx 30 \mathrm{kA}$ and $I_{\max } \approx 60 \mathrm{kA}$, on behalf of $\mathrm{d} I / \mathrm{d} t_{\mid t=0}$ the area within the range of value $0.5 \cdot 10^{12}-2.5 \cdot 10^{12} \mathrm{~A} / \mathrm{s}$ was chosen. 
Table 1: Parameters of pulse shape for $I_{\max }=30 \mathrm{kA}$

\begin{tabular}{|c|c|c|c|c|c|}
\hline $\begin{array}{c}\mathrm{d} I / \mathrm{d} t_{\mid t=0} \\
(\mathrm{~A} / \mathrm{s})\end{array}$ & $\begin{array}{c}I_{\max } \\
(\mathrm{kA})\end{array}$ & $\begin{array}{c}t_{\max } \\
(\mathrm{ns})\end{array}$ & $\begin{array}{c}I_{0} \\
(\mathrm{kA})\end{array}$ & $\begin{array}{c}t_{0} \\
(\mathrm{~ns})\end{array}$ & $\begin{array}{c}t_{1} \\
(\mathrm{~ns})\end{array}$ \\
\hline $0.750 \cdot 10^{12}$ & 30.023 & 67.6 & 33.900 & 71 & 570 \\
\hline $0.923 \cdot 10^{12}$ & 30.031 & 54 & 33.082 & 56.3 & 570 \\
\hline $1.000 \cdot 10^{12}$ & 30.003 & 49.5 & 32.786 & 51.5 & 570 \\
\hline $1.250 \cdot 10^{12}$ & 30.049 & 39.4 & 32.229 & 40.5 & 570 \\
\hline $1.500 \cdot 10^{12}$ & 30.015 & 32.5 & 31.799 & 33.3 & 570 \\
\hline
\end{tabular}

Table 2: Parameters of pulse shape for $I_{\max }=60 \mathrm{kA}$

\begin{tabular}{|c|c|c|c|c|c|}
\hline $\begin{array}{c}\mathrm{d} I / \mathrm{d} t_{\mid t=0} \\
\mathrm{~A} / \mathrm{s}\end{array}$ & $\begin{array}{c}I_{\max } \\
(\mathrm{kA})\end{array}$ & $\begin{array}{c}t_{\max } \\
(\mathrm{ns})\end{array}$ & $\begin{array}{c}I_{0} \\
(\mathrm{kA})\end{array}$ & $\begin{array}{c}t_{0} \\
(\mathrm{~ns})\end{array}$ & $\begin{array}{c}t_{1} \\
(\mathrm{~ns})\end{array}$ \\
\hline $0.923 \cdot 10^{12}$ & 60.056 & 115.15 & 74.213 & 126.3 & 570 \\
\hline $1.000 \cdot 10^{12}$ & 60.029 & 105.05 & 72.765 & 114.3 & 570 \\
\hline $1.250 \cdot 10^{12}$ & 60.006 & 82.020 & 69.630 & 87.5 & 570 \\
\hline $1.500 \cdot 10^{12}$ & 60.044 & 67.878 & 67.8 & 71 & 570 \\
\hline $1.750 \cdot 10^{12}$ & 60.027 & 57.57 & 66.51 & 59.7 & 570 \\
\hline $2.000 \cdot 10^{12}$ & 60.002 & 50 & 65.27 & 51.5 & 570 \\
\hline $2.250 \cdot 10^{12}$ & 60.005 & 43.63 & 64.88 & 45.3 & 570 \\
\hline $2.500 \cdot 10^{12}$ & 60.095 & 39.59 & 64.45 & 40.5 & 570 \\
\hline
\end{tabular}

The last parameter is initial density $\mathrm{N}$, according to the theoretical analysis and in order to achieve the gain maximum, the maximum electron density for carbon has to be $N_{e} \approx 3 \cdot 10^{20}$. To accomplish this requirement, we chose the range $N=0.5 \cdot 10^{17}-5 \cdot 10^{17} \mathrm{~cm}^{-3}$.

\section{Gain evaluation}

The selected parameters for each case served as input data for NPINCH. A very useful output of the calculation was the dens.dat file, which contained a time history of electron and ion density, and electron temperature. This file futher served as input for the FLY and the FLYPAPER code, and by means of these we obtained the time histories of populations on set levels $N_{3}$ for level $n=3$ and $N_{2}$ for level $n=2$ H-like carbon ions. Inversion function $F[3]$ is represented by Equation 3, where the statistical weights $g_{u} / g_{l}$ are for our transition $9 / 4$, and $N_{l}, N_{u}$ presents the lower and upper laser state population densities.

$$
F=1-\left(2.25 \frac{N_{1}}{N_{u}}\right)
$$

The cross-section with corresponding coefficients for our transition $\lambda_{u l}=13.3818 \cdot 10^{-7} \mathrm{~cm}, A_{u l}=$ $5.72 \cdot 10^{10} \mathrm{~s}^{-1}$ can be illustrated as

$$
\begin{aligned}
\sigma_{\text {stim }}= & \frac{\lambda^{3}}{8 \pi c \Delta \lambda / \lambda} A_{u l}= \\
& \frac{1.62 \cdot 10^{4} \cdot(18.2178)^{3} \cdot 5.72 \cdot 10^{10}}{8 \cdot \pi \cdot 2.997925 \cdot 10^{10} \cdot \sqrt{2 \pi}}= \\
& 2.966136 \cdot 10^{-15} \mathrm{~cm}^{2}
\end{aligned}
$$

And finally, $G$ after cross-section substitution can be illustrated as

$$
G=2.966136 \cdot 10^{-15} N_{u} F \mathrm{~cm}^{-1}
$$

For each calculation, we listed the parameter data, information about the maximum value and the time of reaching gain, the pinch time (the time when the maximum of electron density $N_{e}$ is reached).

\section{$4 \quad I_{\max }=30 \mathrm{kA}$}

The results for $I_{\max }=30 \mathrm{kA}$, are in Fig. 1 . The maximum gain value was achieved at $\mathrm{d} I / \mathrm{d} t_{\mid t=0}=$ $1 \cdot 10^{12} \mathrm{~A} / \mathrm{s}$, initial carbon density $1.4 \cdot 10^{17} \mathrm{~cm}^{-3}$ and $\mathrm{G}_{\max } \approx 0.05 \mathrm{~cm}^{-1}$. The calculated data resembled the Gauss function, so they were fitted by this function, and the deviation between the calculated data and the fitted function was minimal. The most information is shown in Fig. 2. The best gain was achieved when the time of reaching current maximum was equal to the pinch time.

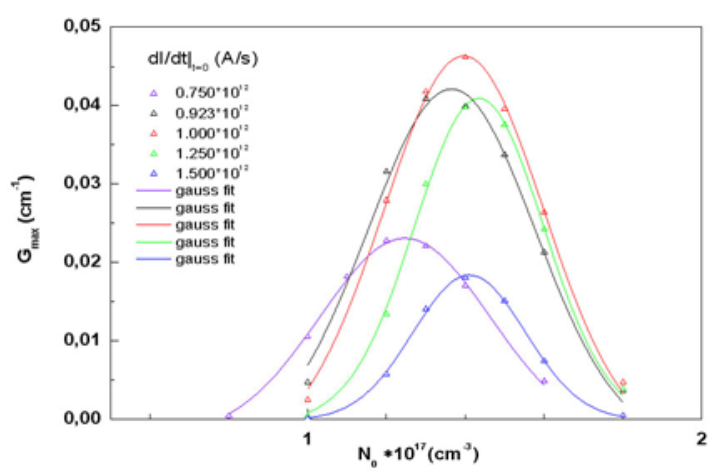

Fig. 1: Maximum $G_{\max }$ against initial density for various current derivations at $I_{\max }=30 \mathrm{kA}$. The data is fitted by the Gauss function

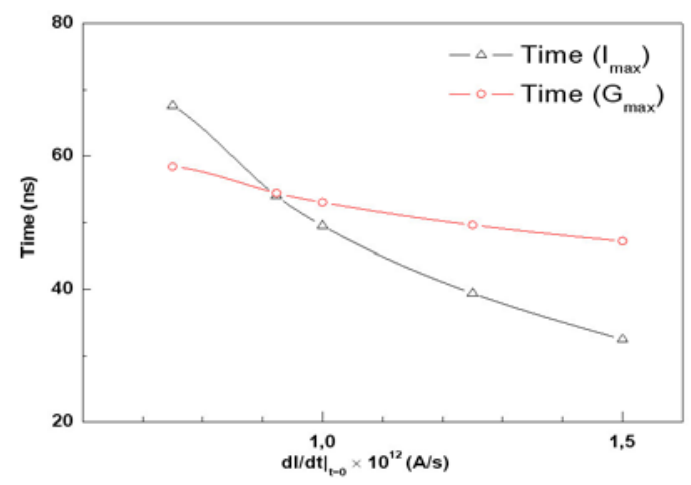

Fig. 2: Comparison of the achieving time of maximum gain $T_{G}$, and current $T_{I}$ in dependence on $\mathrm{d} I / \mathrm{d} t_{\mid t=0}$

\section{$5 \quad I_{\max }=60 \mathrm{kA}$}

For the case where $I_{\max }=60 \mathrm{kA}$ is chosen, the results are shown in the next figure. The maximum gain value 
$G_{\max }$ was reached at $\mathrm{d} I / \mathrm{d} t_{\mid t=0}=2.25 \cdot 10^{12} \mathrm{~A} / \mathrm{s}$, initial carbon density $4.1 \cdot 10^{17} \mathrm{~cm}^{-3}$ and $G_{\max } \approx 3.4 \mathrm{~cm}^{-1}$. This data too was fitted by the Gauss function.

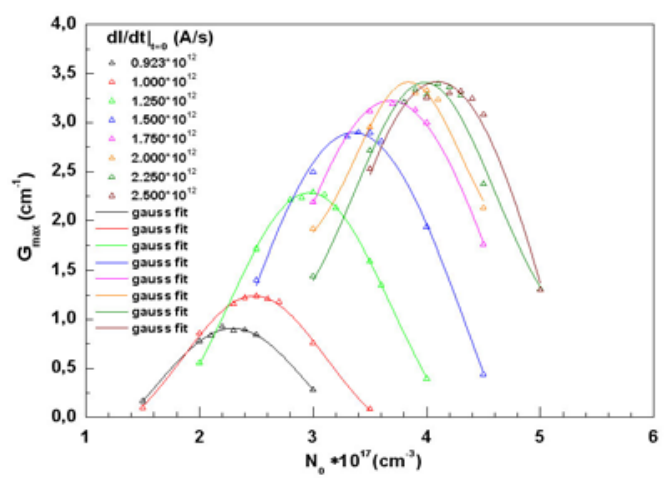

Fig. 3: Maximum $G_{\max }$ against initial density for various current derivations at $I_{\max }=60 \mathrm{kA}$. The data is fitted by the Gauss function

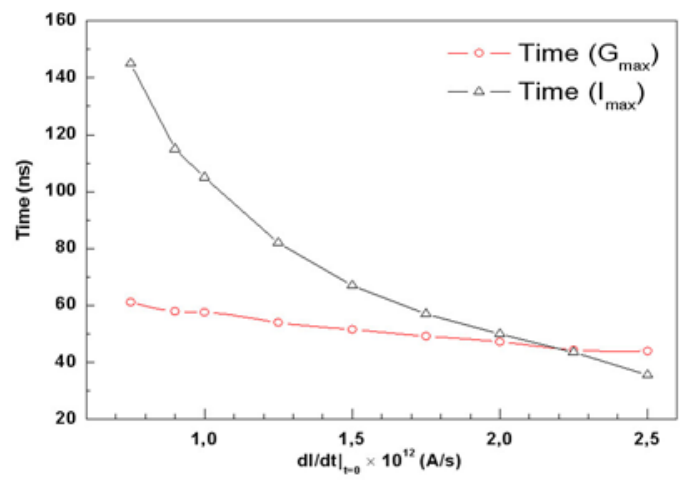

Fig. 4: Comparison of achieving time of maximum gain $T_{G}$ and current $T_{I}$ in dependence on $\mathrm{d} I / \mathrm{d} t_{\mid t=0}$

\section{Example}

Now we can take a closer look at a particular case to show the history of certain variables. The parameters of a capillary discharge $N_{0}=4.0 \cdot 10^{17} \mathrm{~cm}^{-3}$, $\mathrm{d} I / \mathrm{d} t_{\mid t=0}=2 \cdot 10^{12} \mathrm{~A} / \mathrm{s}, r_{0}=1.6 \mathrm{~mm}$ and $I_{\max }=$ $60 \mathrm{kA}$ have been chosen. We will plot the curve for an individual quantity, such as electron density, electron temperature, inversion of population, and gain.

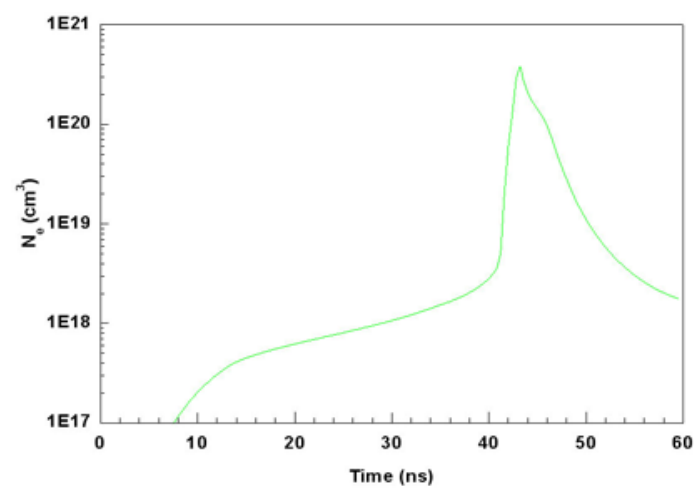

Fig. 5: Behaviour of electron density for parameters $N_{0}=$ $4.0 \cdot 10^{17} \mathrm{~cm}^{-3}, \mathrm{~d} I / \mathrm{d} t_{\mid t=0}=2 \cdot 10^{12} \mathrm{~A} / \mathrm{s}, r_{0}=1.6 \mathrm{~mm}$ and, $I_{\max }=60 \mathrm{kA}$

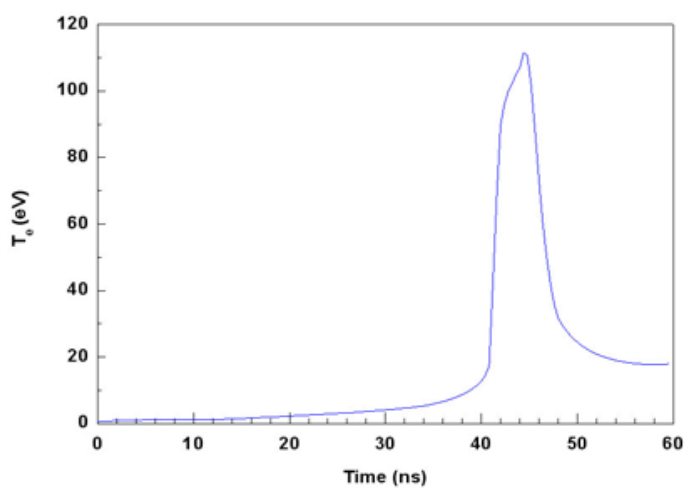

Fig. 6: Behaviour of electron temperature for parameters $N_{0}=4.0 \cdot 10^{17} \mathrm{~cm}^{-3}, \mathrm{~d} I / \mathrm{d} t_{\mid t=0}=2 \cdot 10^{12} \mathrm{~A} / \mathrm{s}, r_{0}=1.6 \mathrm{~mm}$, and $I_{\max }=60 \mathrm{kA}$

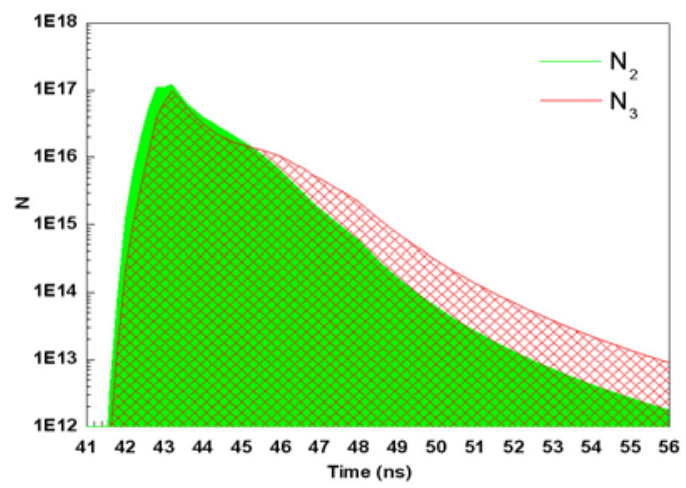

Fig. 7: Behaviour of the population on levels $n=2$ a $n=3$ for parameters $N_{0}=4.0 \cdot 10^{17} \mathrm{~cm}^{-3}, \mathrm{~d} I / \mathrm{d} t_{\mid t=0}=$ $2 \cdot 10^{12} \mathrm{~A} / \mathrm{s}, r_{0}=1.6 \mathrm{~mm}$, and $I_{\max }=60 \mathrm{kA}$

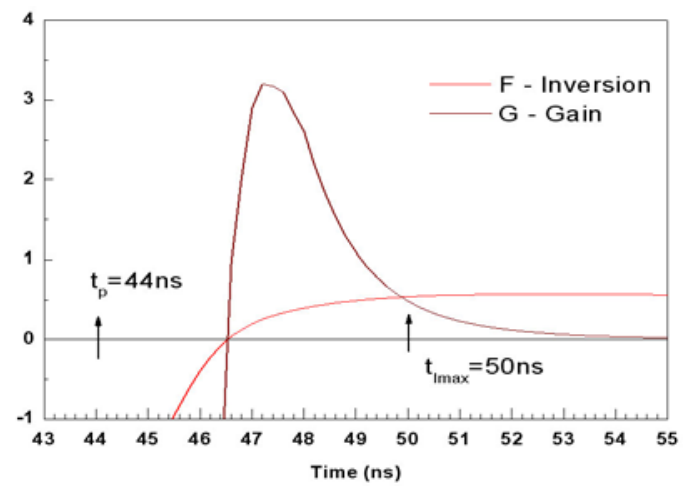

Fig. 8: Behaviour of inversion function $F$ and gain $\left(\mathrm{cm}^{-1}\right)$. In addition, the figure contains a marked pinch time $t_{p}=$ $44 \mathrm{~ns}$ and time of reaching current maximum $t_{I_{\max }}=55$ for parameters $N_{0}=4.0 \cdot 10^{17} \mathrm{~cm}^{-3}, \mathrm{~d} I / \mathrm{d} t_{\mid t=0}=2 \cdot 10^{12} \mathrm{~A} / \mathrm{s}$, $r_{0}=1.6 \mathrm{~mm}$ and $I_{\max }=60 \mathrm{kA}$

Figures 6 and 7 demonstrate the behaviour of electron density and temperature. They show pinch time $t_{p}=44 \mathrm{~ns}, N_{\text {emax }}=3.8 \cdot 10^{20} \mathrm{~cm}^{-3}, T_{\text {emax }}=110 \mathrm{eV}$. After that, a rapid drop in these quantities follows due to adiabatic expansion. The electron density drops by nearly two orders in 5 ns and the electron temperature drops by nearly $90 \mathrm{eV}$. These are ideal conditions for creating population inversion at the desired levels. 
Figure 8 shows that at time $46.5 \mathrm{~ns}, 2.5 \mathrm{~ns}$ after pinch time, both the inversion function and gain had an above zero value. At time $47.2 \mathrm{~ns}$, the gain maximum $G_{\max }=3.2 \mathrm{~cm}^{-1}$ is reached, and just about one ns later the inversion function also reaches its maximum and starts to fall steadily. However, the gain begins to drop very rapidly, due to rapid density decay of the whole mass (both electrons and ions, which are lasing) along to axix. It follows from the gain behaviour that the hypothetical laser pulse could be 2 ns in length.

How does the initial density influence the pinch behaviour? With increasing initial density the maximum density also increases. At the same time, the pich time is delayed. The effect is totally contrary to the electron temperature. As the initial density increases the maximum electron temperature value decreases.

\section{Conclusion}

The optimal conditions for achieving maximum gain for initial density and electric current pulse shape have been obtained for two different current maxima, $I_{\max }=30 \mathrm{kA}, I_{\max }=60 \mathrm{kA}$ and capillary radius $r=1.6 \mathrm{~mm}$. Figures 2 and 4 show clearly that the time of achieving the current maximum and the pinch time have to be synchronized for optimal current differentiation at zero time, so that both times will be almost identical.

$G_{\max }=0.05 \mathrm{~cm}^{-1}$ was achieved at $N_{0}=1.4$ $10^{17} \mathrm{~cm}^{-3}, \mathrm{~d} I / \mathrm{d} t_{\mid t=0}=1 \cdot 10^{12} \mathrm{~A} / \mathrm{s}, r_{0}=1.6 \mathrm{~mm}$ and $I_{\max }=30 \mathrm{kA}$.
$G_{\max }=3.4 \mathrm{~cm}^{-1}$ was achieved at $N_{0}=4.1$. $10^{17} \mathrm{~cm}^{-3}, \mathrm{~d} I / \mathrm{d} t_{\mid t=0}=2.25 \cdot 10^{12} \mathrm{~A} / \mathrm{s}, r_{0}=1.6 \mathrm{~mm}$ and $I_{\max }=60 \mathrm{kA}$. The assumed pulse length is $2 \mathrm{~ns}$.

\section{Acknowledgement}

The research described in this paper was supervised by Ing. Pavel Vrba, CSc., IPP AS CR in Prague, and was supported by the Czech Grant Agency under grant No. 202/08/057.

\section{References}

[1] Rocca, J. J.: Table-top soft $x$-ray lasers, Review of Scientific Instruments, 1999, vol. 70, no. 10.

[2] Razinkova, T. L., Sasorov, P. V.: Program NPINCH na počítání dynamiky z-pinče, Moskva, 1998.

[3] Elton, R. C.: X-Ray Laser, Academic Press, New York, 1990.

[4] Koláček, K. et al.: 10th ICXRL2006, Berlin, Germany (poster - 14_09_Kolacek.pdf).

Jakub Hübner

E-mail: bukajus@centrum.cz

Dept. of Physical Electronics

Faculty of Nuclear Science and Physical Engineering

Czech Technical University

Břehová 7, 11519 Prague 1, Czech Republic 